\title{
On the stability of thermonuclear shell sources in stars
}

\author{
S.-C. Yoon, N. Langer, and M. van der Sluys
}

\author{
Astronomical Institute, Utrecht University, Princetonplein 5, 3584 CC, Utrecht, The Netherlands \\ e-mail: s.c.yoon@astro.uu.nl
}

Received 9 February 2004 / Accepted 1 June 2004

\begin{abstract}
We present a quantitative criterion for the thermal stability of thermonuclear shell sources. We find that the thermal stability of shell sources depends on exactly three factors: they are more stable when they are geometrically thicker, less degenerate and hotter. This confirms and unifies previously obtained results in terms of the geometry, temperature and density of the shell source, by a simplified but quantitative approach to the physics of shell nuclear burning. We present instability diagrams in the temperature-density plane for hydrogen and helium shell burning, which allow a simple evaluation of the stability conditions of such shell sources in stellar models. The performance of our stability criterion is demonstrated in various numerical models: in a $3 M_{\odot}$ AGB star, in helium accreting CO white dwarfs, in a helium white dwarf which is covered by a thin hydrogen envelope, and in a $1.0 M_{\odot}$ giant.
\end{abstract}

Key words. instabilities - nuclear reactions, nucleosynthesis, abundances - stars: interiors - stars: AGB and post-AGB stars: white dwarfs

\section{Introduction}

Thermonuclear fusion is the main source of energy in stars. To use fusion as main energy supply over long time scales requires extreme stability, since the thermonuclear reaction rates are sensitive functions of the temperature. I.e., a slight temperature increase may enhance the nuclear burning rate drastically; if this results in an even higher temperature, a thermal runaway occurs. In most stars, such a runaway does not occur, since an increased energy output due to an enhancement of the nuclear burning rate leads to an overpressure and thus to an expansion of the burning region (Kippenhahn \& Weigert 1990). However, two different circumstances have been shown to be able to prevent such a pressure increase.

The first one is electron degeneracy. If nuclear burning starts in a degenerate gas, the increased energy production will lead to a higher temperature. But as - for complete degeneracy - the pressure does not depend on the temperature, it remains constant. This situation is realized during the core helium flash in low mass stars, at carbon ignition in Chandrasekhar-mass $\mathrm{CO}$ white dwarfs, i.e. in a Type Ia supernova explosion, and in strong nova shell flashes.

The second circumstance is a strong geometrical confinement of the burning region. As discovered by Schwarzshild \& Härm (1965) and Weigert (1966), geometrically thin helium shell sources in red giants can become unstable. Since the local gravity in a thin shell source remains constant even when it expands in response to an increased energy production, hydrostatic equilibrium will enforce a constant pressure in the shell. The thin shell instability is prone to occur in nuclear shell sources around compact cores, as the small scale heights induced by the compactness of the core ensure the geometrical confinement of the shells. Examples are thermal pulses in asymptotic giant branch stars (e.g. Iben \& Renzini 1983; Lugaro et al. 2003), hydrogen or helium shells on white dwarfs (e.g. Fujimoto 1982a,b; Cassisi et al. 1998; Langer et al. 2002; Yoon et al. 2004), and X-ray bursts on neutron stars (e.g. Fujimoto et al. 1981; Taam \& Woosley 1996).

In principle, both destabilizing circumstances may occur together. In central burning regions, however, the degeneracy aspect dominates, as gravity and thus pressure will not be constant in an expanding core. In nuclear shell sources, degeneracy and geometrical confinement may simultaneously contribute to the instability of shell sources. Among various authors investigating the stability of nuclear shell sources (e.g. Giannone \& Weigert 1967; Unno 1970; Dennis 1971; Härm \& Schwarzshild 1972; Stothers \& Chin 1972; Sackmann 1977), Kippenhahn \& Weigert (1990) developed a semi-quantitative understanding of the relevance of both destabilizing factors.

The purpose of this paper is to develop the insight into the shell stability mechanism further such that a quantitative tool is obtained, which makes it possible to assess the thermal stability of shell sources in numerical stellar models. This will allow a physical interpretation of fluctuating nuclear shell sources found in non-linear time-dependent stellar evolution calculations. As described in Sect. 2, we essentially follow the method of Giannone \& Weigert (1967), but assume homology in the region of the shell source as in Kippenhahn \& Weigert (1990). In Sect. 3, we derive thermodynamic parameters for partial degenerate conditions, which we use in Sect. 4 to formulate 
and investigate the stability criterion in idealized situations. In Sect. 5, we apply the criterion to various numerical stellar models, and give a summary and discussion of our results in Sect. 6.

\section{Secular behavior of shell burning}

\subsection{Gravothermal specific heat}

Let us consider a shell source with a geometrical thickness $D$. The mass of the shell source is given as $\Delta M_{\mathrm{s}}=\int_{r_{0}}^{r_{\mathrm{s}}} 4 \pi r^{2} \rho \mathrm{d} r$, where $r_{0}$ is the radius of the bottom of the shell source and $r_{\mathrm{s}}=r_{0}+D$, its upper boundary. Assuming $r_{0}$ is constant, the relation between density and radius perturbation is given from $\mathrm{d} \Delta M_{\mathrm{s}}=0$ as

$\frac{\delta \rho}{\rho}=-\frac{3}{3 D / r_{\mathrm{s}}-3\left(D / r_{\mathrm{s}}\right)^{2}+\left(D / r_{\mathrm{s}}\right)^{3}} \frac{\delta r_{\mathrm{s}}}{r_{\mathrm{s}}}$

(Huang \& Yu 1998). If we assume that the matter in the shell source expands or contracts homologously, we have $\delta P / P=$ $-4 \delta r / r$ from the hydrostatic equation and we obtain:

$\frac{\delta P}{P}=\alpha_{\mathrm{s}} \frac{\delta \rho}{\rho}$

where

$\alpha_{\mathrm{s}}=\frac{4}{3}\left(3 D / r_{\mathrm{s}}-3\left(D / r_{\mathrm{s}}\right)^{2}+\left(D / r_{\mathrm{s}}\right)^{3}\right)$.

Kippenhahn \& Weigert's value $\alpha_{\mathrm{s}}=4 D / r_{\mathrm{s}}$ is restored when $D / r_{\mathrm{s}} \ll 1$, while it becomes $4 / 3$ with $D / r_{\mathrm{s}}=1$, which is the case when the whole star contracts or expands homologously.

Using the thermodynamic relation $\delta \rho / \rho=\alpha_{P} \delta P / P-$ $\alpha_{T} \delta T / T$, it follows from Eq. (2) that

$\frac{\delta P}{P}=\frac{\alpha_{\mathrm{s}} \alpha_{T}}{\alpha_{\mathrm{s}} \alpha_{P}-1} \frac{\delta T}{T}, \quad \frac{\delta \rho}{\rho}=\frac{\alpha_{T}}{\alpha_{\mathrm{s}} \alpha_{P}-1} \frac{\delta T}{T}$

where $\alpha_{P}=(\partial \ln \rho / \partial \ln P)_{T}$ and $\alpha_{T}=-(\partial \ln \rho / \partial \ln T)_{P}$ (Kippenhahn \& Weigert 1990). As we will see in the next section, $\alpha_{T}$ and $\alpha_{P}$ are functions of the degree of degeneracy and are not constant over a shell source. However, since we are interested in the mean properties of a shell source in this study, we will assume that, like the density and the pressure perturbation, the temperature change is also homologous in the shell, and mean values over the shell source will be used for $\alpha_{P}$ and $\alpha_{T}$.

The heat perturbation in the shell source can be obtained from the first law of thermodynamics as shown by Kippenhahn $\&$ Weigert (1990):

$\delta q=c^{*} \delta T$

with

$c^{*}:=c_{\mathrm{P}}\left(1-\nabla_{\mathrm{ad}} \frac{\alpha_{\mathrm{s}} \alpha_{T}}{\alpha_{\mathrm{s}} \alpha_{P}-1}\right)$,

where $c_{P}$ denotes the specific heat at constant pressure and $\nabla_{\mathrm{ad}}=(\mathrm{d} \ln T / \mathrm{d} \ln P)_{\mathrm{ad}}$.

The quantity $c^{*}$ is called the gravothermal specific heat (Kippenhahn \& Weigert 1990). When the heat $\delta q$ is added to the shell source, more energy than $\delta q$ is consumed by the expansion work if $c^{*}<0$, thereby decreasing the internal energy. However, if $c^{*}>0$, the additional energy heats up the matter and thus favors instability.

\subsection{The stability criterion}

The gravothermal specific heat alone does not describe the thermal reaction of a shell source, as it is also influenced by its thermal interaction with its surroundings. The energy conservation in a star is expressed by the following equation:

$\frac{\partial L_{r}}{\partial M_{r}}=\epsilon_{\mathrm{N}}-\frac{\mathrm{d} q}{\mathrm{~d} t}$

For a shell source with thickness $D=r_{\mathrm{s}}-r_{0}$ and mass $\Delta M_{\mathrm{s}}$, integration over the burning shell gives

$L_{r_{\mathrm{s}}}-L_{r_{0}}=L_{\mathrm{N}}-L_{\mathrm{g}}$.

$L_{r_{\mathrm{s}}}$ and $L_{r_{0}}$ are the luminosities at $r=r_{\mathrm{s}}$ and $r=r_{0}$, respectively. $L_{\mathrm{N}}$ is the luminosity due to the nuclear burning in the shell: $L_{\mathrm{N}}=\int_{\Delta M_{\mathrm{s}}} \epsilon_{\mathrm{N}} \mathrm{d} M_{r}$ where $\epsilon_{\mathrm{N}}$ is the nuclear energy generation rate in the shell source. $L_{\mathrm{g}}$ is defined as $\int_{\Delta M_{\mathrm{s}}} \frac{\mathrm{d} q}{\mathrm{~d} t} \mathrm{~d} M_{r}$. In AGB stars or accreting white dwarfs the main contribution to $L_{\mathrm{g}}$ is due to the gravitational energy release by contraction. This term is not significant compared to $L_{\mathrm{N}}$ in general, and we will assume that the shell source is initially in a stationary state (i.e., $\mathrm{d} q / \mathrm{d} t=0$ ).

Perturbing Eq. (8) yields

$\delta L_{r_{\mathrm{s}}}=\Delta M_{\mathrm{s}} \delta \epsilon_{\mathrm{N}}-\Delta M_{\mathrm{s}} \frac{\mathrm{d} \delta q}{\mathrm{~d} t}$

where $L_{r_{0}} \ll L_{r_{\mathrm{s}}}$ has been assumed. With the use of Eqs. (2)-(6) and assuming radiative heat transfer, i.e.,

$\frac{\partial T}{\partial M_{r}}=-\frac{3}{64 \pi^{2} a c} \frac{\kappa L_{r}}{r^{4} T^{3}}$

we obtain the following equation for the temperature perturbation $\theta=\delta T / T$ :

$\tau_{\mathrm{th}} \dot{\theta}=\sigma \theta$,

with

$\tau_{\mathrm{th}}=\frac{\Delta M_{\mathrm{s}} T c_{\mathrm{P}}}{L_{r_{\mathrm{s}}}}$

and

$\sigma=\frac{v-4+\kappa_{\mathrm{T}}+\frac{\alpha_{T}}{\alpha_{\mathrm{s}} \alpha_{P}-1}\left(\lambda+\alpha_{\mathrm{s}}+\kappa_{\rho}\right)}{c^{*} / c_{p}}$.

Here, $\kappa_{\mathrm{T}}=(\partial \ln \kappa / \partial \ln T)_{\rho}, \kappa_{\mathrm{P}}=(\partial \ln \kappa / \partial \ln \rho)_{T}, v=$ $(\partial \ln \epsilon / \partial \ln T)_{\rho}$, and $\lambda=(\partial \ln \epsilon / \partial \ln \rho)_{T}$. The quantity $\tau_{\text {th }}$ defined in Eq. (11) corresponds to the thermal time scale of the shell source. Note that Eq. (10) is essentially the same as Eq. (16) in Giannone \& Weigert (1967), for the case that the assumption of homology in the shell source is adopted in their analysis.

When $\sigma>0$ the shell source becomes thermally unstable and the perturbation growth time scale becomes

$\tau_{\text {growth }}=\tau_{\text {th }} / \sigma$.

The larger the value of $\sigma$, the more rapidly the thermal instability grows. If $\sigma<0$, nuclear burning in a shell source is thermally stable, and, upon a temperature increase, the temperature 
drops faster for larger $\sigma$. Therefore, $\sigma$ can serve as a measure of the susceptibility of a shell source to thermal instability.

For the following discussions, let us define the numerator in Eq. (12) as $\sigma_{\mathrm{E}}$ such that $\sigma=\sigma_{\mathrm{E}} /\left(c^{*} / c_{P}\right)$ and

$\sigma_{\mathrm{E}}=v-4+\kappa_{\mathrm{T}}+\frac{\alpha_{T}}{\alpha_{\mathrm{s}} \alpha_{P}-1}\left(\lambda+\alpha_{\mathrm{s}}+\kappa_{\rho}\right)$.

The first 3 terms in the above equation are related to the temperature perturbation and the remaining terms to the density perturbation.

For a given $c^{*}$, i.e. a given equation of state, the stability of a shell source is determined by the sign of $\sigma_{\mathrm{E}}$. Physically, $\sigma_{\mathrm{E}}>0$ means that the additional energy production in response to a positive temperature perturbation exceeds the additional energy loss by radiation. In other words, for $\delta T>0$, we have $\delta q>0$ if $\sigma_{\mathrm{E}}>0$, in which case a shell source is stable if $c^{*}<0$, since the additional heat is consumed mostly for the expansion work of the shell source as discussed in Sect. 2.1. If $\sigma_{\mathrm{E}}<0$, the additional energy loss by radiation is larger than the additional energy production (i.e., $\delta q<0$ ) in response to $\delta T>0$. If $c^{*}<0$ and $\sigma_{\mathrm{E}}<0$, this results in further increase in the temperature perturbation since the net energy loss is compensated by the contraction of the system, increasing the internal energy of the shell source. However, this instability mode has little relevance as in stars $\sigma_{\mathrm{E}}$ hardly becomes negative if $c^{*}<0$, as we will see in Sect. 3.1. If $c^{*}>0$ and $\sigma_{\mathrm{E}}<0$, the net energy loss results in a decrease in the internal energy and stable burning is established.

In the following sections, we will investigate the possible modes of stability/instability of a shell source in a more quantitative way.

\section{Physical conditions for stability/instability}

To discuss the stability of shell source in realistic stars, it is necessary to understand the behaviour of the thermodynamic quantities such as $\alpha_{P}, \alpha_{T}$ and $c^{*}$ in various equation-of-state regimes.

For non-relativistic partially degenerate conditions, electron density and pressure are given by

$n_{\mathrm{e}}=\frac{\rho}{\mu_{\mathrm{e}} m_{\mathrm{H}}}=\frac{4 \pi}{h^{3}}\left(2 m_{\mathrm{e}} k T\right)^{3 / 2} F_{1 / 2}(\eta)$

and

$P_{\mathrm{e}}=\frac{\rho k T}{\mu_{\mathrm{e}} m_{\mathrm{H}}} \frac{2 F_{3 / 2}(\eta)}{3 F_{1 / 2}(\eta)}$

where $F_{3 / 2}$ and $F_{1 / 2}$ are the Fermi-Dirac functions and $\eta:=$ $\psi / k T$ is the degeneracy parameter (e.g. Clayton 1968). The equation of state is thus given as:

$P=P_{\mathrm{g}}+P_{\mathrm{r}}=\frac{\rho k T}{\mu_{\mathrm{I}} m_{\mathrm{H}}}+\frac{\rho k T}{\mu_{\mathrm{e}} m_{\mathrm{H}}} \frac{2 F_{3 / 2}(\eta)}{3 F_{1 / 2}(\eta)}+\frac{1}{3} a T^{4}$.

With the definition of $\beta:=P_{\mathrm{g}} / P$, the above equation can be rewritten as

$F(\eta)+\frac{\mu_{\mathrm{e}}}{\mu_{\mathrm{I}}}=\frac{\mu_{\mathrm{e}} m_{\mathrm{H}} \beta P}{\rho k T}$, where $F:=2 F_{3 / 2} / 3 F_{1 / 2}$. Differentiating this equation, we get

$\frac{F}{F+\mu_{\mathrm{e}} / \mu_{\mathrm{I}}} \mathrm{d} \ln F=\frac{1}{\beta} \mathrm{d} \ln P-\frac{4-3 \beta}{\beta} \mathrm{d} \ln T-\mathrm{d} \ln \rho$

where $(\partial \ln \beta / \partial \ln P)_{T}=(1-\beta) / \beta$ and $(\partial \ln \beta / \partial \ln T)_{P}=4(\beta-$ $1) / \beta$ were used. From Eq. (19) and by differentiating Eq. (15), we can obtain $\alpha_{P}$ and $\alpha_{T}$ as a function of $\eta$ :

$\alpha_{P}=\frac{\frac{1}{\beta} \frac{\mathrm{d} \ln F_{1 / 2}}{\mathrm{~d} \eta}}{\frac{\mathrm{d} \ln F_{1 / 2}}{\mathrm{~d} \eta}+\frac{F}{F+\mu_{\mathrm{e}} / \mu_{\mathrm{I}}} \frac{\mathrm{d} \ln F}{\mathrm{~d} \eta}}$

and

$\alpha_{T}=\frac{\frac{4-3 \beta}{\beta} \frac{\mathrm{d} \ln F_{1 / 2}}{\mathrm{~d} \eta}-\frac{3}{2} \frac{F}{F+\mu_{\mathrm{e}} / \mu_{\mathrm{I}}} \frac{\mathrm{d} \ln F}{\mathrm{~d} \eta}}{\frac{\mathrm{d} \ln F_{1 / 2}}{\mathrm{~d} \eta}+\frac{F}{F+\mu_{\mathrm{e}} / \mu_{\mathrm{I}}} \frac{\mathrm{d} \ln F}{\mathrm{~d} \eta}}$.

When $\beta=1$, we get $\alpha_{P}=\alpha_{T}=1$ for an ideal gas $(\eta \rightarrow-\infty$, Fig. 1), and $\alpha_{P}=0.6$ and $\alpha_{T}=0$ for complete degeneracy $(\eta \rightarrow$ $\infty)$. In the case of $\beta \neq 0$, we have $\alpha_{P}=1 / \beta$ and $\alpha_{T}=(4-3 \beta) / \beta$ in a non-degenerate gas (cf. Kippenhahn \& Weigert 1990), since $F(\eta) \rightarrow 1$ and $\mathrm{d} \ln F / \mathrm{d} \eta \rightarrow 0$ when $\eta \rightarrow-\infty$.

The specific heat at constant pressure is given by $c_{P}=$ $\left(\frac{\partial u}{\partial T}\right)_{P}-\frac{P}{\rho^{2}}\left(\frac{\partial \rho}{\partial T}\right)_{P}$ (Kippenhahn \& Weigert 1990). From $u=$ $\left(\frac{3}{2} P_{\mathrm{g}}+3 P_{\mathrm{r}}\right) / \rho=\left(3-\frac{3}{2} \beta\right) P / \rho$, we have

$c_{P}=\frac{P}{\rho T}\left[\left(\frac{5}{2}+\frac{3}{2}(1-\beta)\right) \alpha_{T}+6(1-\beta)\right]$

and

$\nabla_{\mathrm{ad}}=\frac{P \alpha_{T}}{\rho T c_{P}}=\frac{\alpha_{T}}{\left(\frac{5}{2}+\frac{3}{2}(1-\beta)\right) \alpha_{T}+6(1-\beta)}$.

Since $P / \rho T$ in Eq. (22) can also be given as a function of $\eta$ from Eq. (18), the gravothermal specific heat $c^{*}$ can be obtained as a function of $\eta$ for a specified $\beta$.

The value of $\beta$ can be obtained as a function of $\eta$ at a given temperature from the equation of state. In Fig. 1a, we show $\beta$ as a function of $\eta$ for 4 different temperatures which may be relevant to the helium shell burning. $\mu_{\mathrm{e}}=2$ and $\mu_{\mathrm{e}} / \mu_{\mathrm{I}}=0.38$ have been assumed. In this temperature range, $\beta$ reaches 1 when $\eta \gtrsim 0$ since the gas pressure becomes dominant.

As discussed in Sects. 3.1-4, the values of $\alpha_{T}$ and $\alpha_{P}$ and the sign of $c^{*}$ affect the stability of a shell source critically. Figures $1 \mathrm{~b}$ and $1 \mathrm{c}$ show $\alpha_{P}$ and $\alpha_{T}$ as a function of $\eta$, obtained using the values of $\beta$ given in Fig. 1a. The case when the radiation pressure is neglected (i.e., $\beta=1$ ) is also shown in these figures. Since changes in the total pressure become more sensitive to changes in temperature than in density as radiation pressure becomes significant, we find larger values of $\alpha_{P}$ and $\alpha_{T}$ when radiation pressure is considered than in the case of $\beta=1$. Since $\nabla_{\text {ad }}$ varies little compared to $\alpha_{P}$ and $\alpha_{T}$, the sign of $c^{*}$ is mainly determined by the sign of $\alpha_{\mathrm{s}} \alpha_{P}-1$ (see Eq. (6)).

Figure 2 shows the lines of zero gravothermal specific heat $c^{*}$, in the plane spanned by the degeneracy parameter $\eta$ and the relative thickness of the shell source $D / r_{\mathrm{s}}$, for 4 different temperatures. These lines have been determined using 
(a)

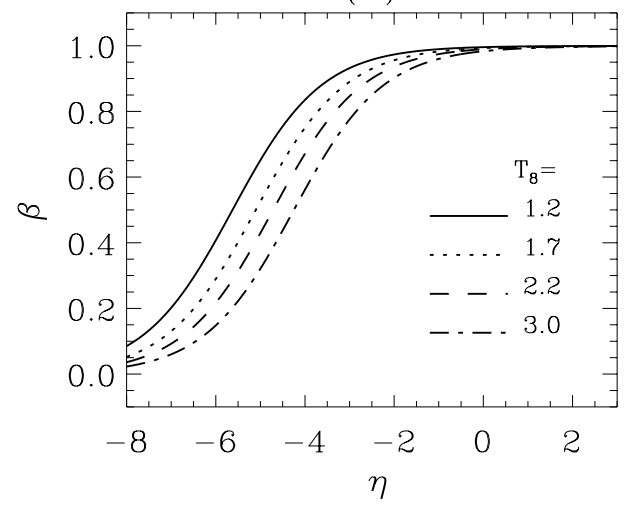

(c)

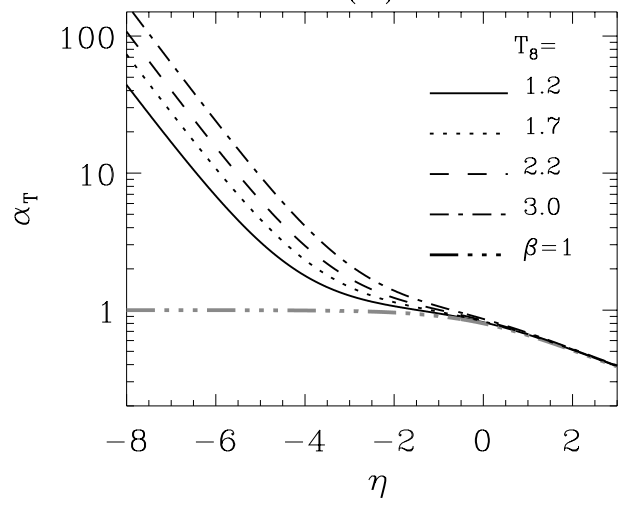

(b)

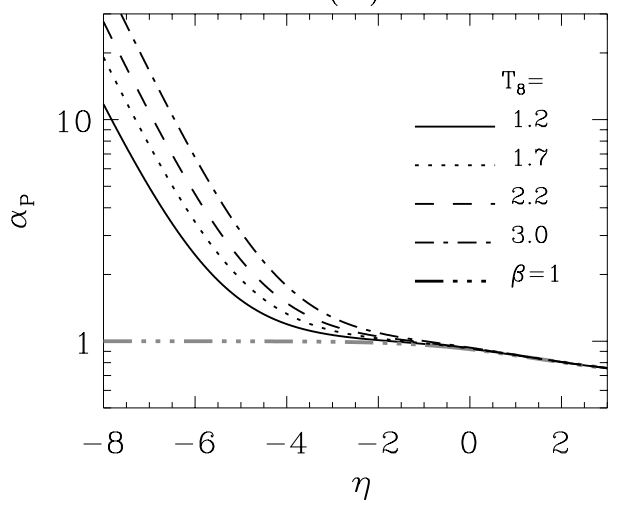

(d)

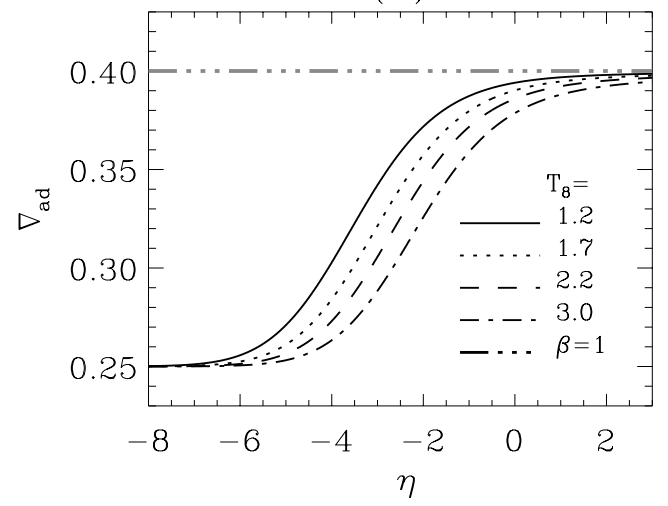

Fig. 1. Thermodynamic quantities as a function of the degeneracy parameter $\eta$ for 4 different temperatures as indicated. Here, $T_{8}$ denotes temperature in units of $10^{8} \mathrm{~K}$. As a reference, the case of $\beta=1$ is also shown for $\alpha_{P}, \alpha_{T}$ and $\nabla_{\mathrm{ad}}$. a) - Ratio of gas pressure to total pressure, $\beta$, as a function of the degeneracy parameter. b), c) $-\alpha_{P}\left(:=(\partial \ln \rho / \partial \ln P)_{T}\right)$ and $\alpha_{T}\left(:=(\partial \ln \rho / \partial \ln T)_{P}\right)$ calculated from Eqs. (20) and (21) using $\beta$ given in a). d) $-\nabla_{\mathrm{ad}}\left(:=(\partial \ln T / \partial \ln P)_{\mathrm{ad}}\right)$ calculated from Eq. (23) using $\beta$ and $\alpha_{T}$ given in a) and c) respectively.

the values of $\beta, \alpha_{T}, \alpha_{P}$ and $\nabla_{\text {ad }}$ given in Fig. 1 . For $\beta=1$, the thickness of the shell source should be larger than $0.37 r_{\mathrm{s}}$ for the gravothermal specific heat to be negative in the non-degenerate region $(\eta \lesssim-2)$. As the electron degeneracy becomes more significant, the minimum thickness which gives $c^{*}<0$ becomes larger.

In the non-degenerate region, $c^{*}$ remains negative for much smaller shell thicknesses when the effect of the radiation pressure is considered. This is due to the fact that $\alpha_{P}$ and $\alpha_{T}$ become large when radiation pressure is significant. Since shell sources are generally stable when $c^{*}<0$ as discussed in Sect. 3.1, this indicates that radiation pressure serves as a stabilizing factor for a shell source. The stabilizing effect of radiation pressure, which has already been discussed by many authors (e.g. Dennis 1971; Stothers \& Chin 1972; Sackmann 1977), is due to the fact that a small increase in temperature induces considerable expansion of the shell source when radiation pressure is significant (cf. Eq. (4)).

\subsection{The case of $c^{*}<0$}

When $c^{*}<0$, the following two conditions are fulfilled from Eq. (6):

$\alpha_{\mathrm{s}} \alpha_{P}-1>0$ and $\alpha_{\mathrm{s}} \alpha_{P}-1<\nabla_{\mathrm{ad}} \alpha_{\mathrm{s}} \alpha_{T}$.
The latter condition can be rewritten as $\alpha_{P}-\nabla_{\mathrm{ad}} \alpha_{T}<1 / \alpha_{\mathrm{s}}$. In a non-degenerate gas (i.e., $\mathrm{d} \ln F / \mathrm{d} \eta \rightarrow 0$ ), we have $\alpha_{P}-\nabla_{\mathrm{ad}} \alpha_{T} \rightarrow 0.75$ from Eqs. (20), (21) and (23), while we have $\alpha_{P}-\nabla_{\mathrm{ad}} \alpha_{T} \rightarrow 0.6$ in a completely degenerate gas where $\alpha_{P}=0.6$ and $\alpha_{T}=0$. It follows that the inequality $\alpha_{\mathrm{s}} \alpha_{P}-1<\nabla_{\mathrm{ad}} \alpha_{\mathrm{s}} \alpha_{T}$ is always satisfied for all realistic values of $\alpha_{\mathrm{s}}$. Therefore, the condition $c^{*}<0$ reduces to $\alpha_{\mathrm{s}} \alpha_{P}-1>0$. With this condition, the term $\alpha_{T} /\left(\alpha_{\mathrm{s}} \alpha_{P}-1\right)$ in Eq. (14) is positive, and it is generally $\sigma_{\mathrm{E}}>0$ since $v$ dominates over $\kappa_{T}$ and $\kappa_{\rho}$ in most cases.

For example, let us consider a helium shell source powered by the $3 \alpha$-reaction. Figure 3 shows $v$ as a function of temperature, calculated from the $3 \alpha$ reaction rate given by Harris et al. (1983). The density dependence $\lambda$ is equal to 2 regardless of temperature. In AGB stars or in accreting white dwarfs, the temperature of the helium shell source varies according to the core mass or the accretion rate within the range $1<T / 10^{8} \mathrm{~K}<$ 3 , giving $v=40 \cdots 12$. Figure 4 shows the contour levels of $\kappa_{T}$ and $\kappa_{\rho}$ in the $T-\rho$ plane, calculated from the OPAL opacity table by Iglesias \& Rogers (1996). The helium abundance has been assumed to be 0.66 in this calculation since the energy generation reaches its maximum around this value in a helium shell source. This figure shows that $\kappa_{T}>-1$ and $\kappa_{\rho} \ll 1$ in the given temperature and density range. Therefore, $\sigma_{\mathrm{E}}>0$ is guaranteed as long as $\alpha_{T} /\left(\alpha_{\mathrm{s}} \alpha_{P}-1\right)>0$. The situation is very 


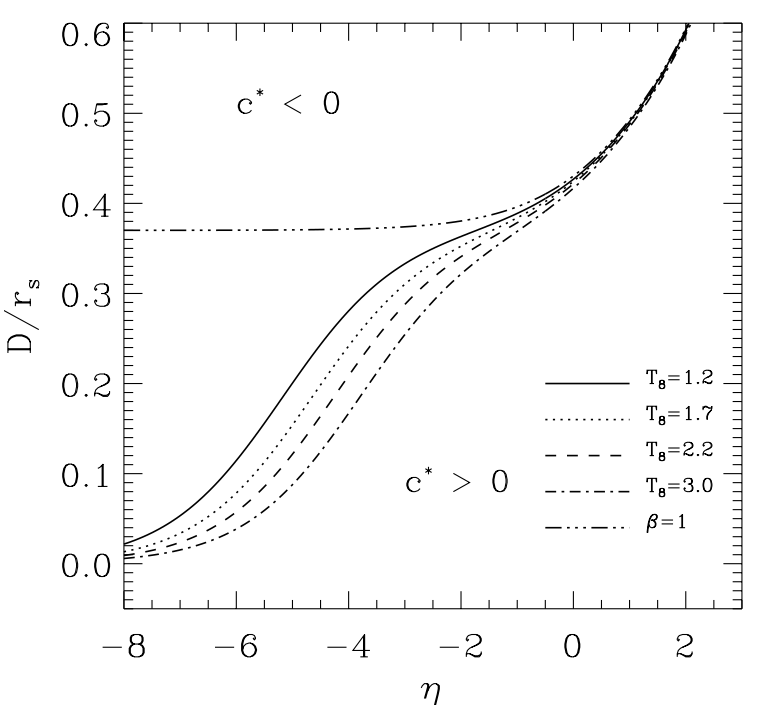

Fig. 2. Lines of vanishing gravothermal specific heat $c^{*}$ in the plane spanned by the degeneracy parameter $\eta$ and the relative thickness of the shell source $D / r_{\mathrm{s}}$, for four different temperatures. These lines are computed by using the thermodynamic quantities given in Fig. 1 . The line labeled $\beta=1$ shows the case where radiation pressure is neglected.

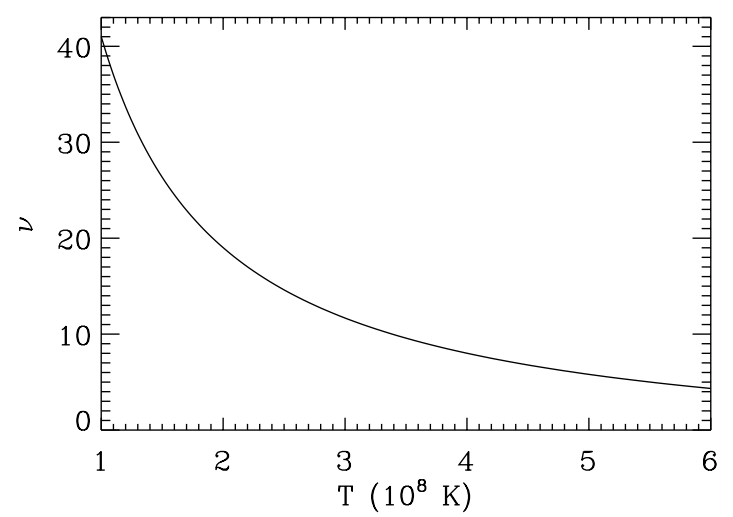

Fig. 3. The logarithmic derivative of the $3 \alpha$ reaction with respect to temperature: $v=\left(\partial \ln \epsilon_{3 \alpha} / \partial \ln T\right)_{\rho}$. The reaction rate given by Harris et al. (1983) has been used in the calculation.

similar even in hydrogen shell sources if they are powered by the CNO cycle. In conclusion, the shell source is generally stable (i.e., $\sigma<0$ ) under the condition $c^{*}<0$, i.e. for negative gravothermal specific heat.

\subsection{The case of $c^{*}>0$}

For $c^{*}>0$, we have

$\alpha_{\mathrm{s}} \alpha_{P}-1<0$.

In this case, we have $\alpha_{T} /\left(\alpha_{\mathrm{s}} \alpha_{P}-1\right)<0$. In general, a shell source is prone to thermal instability when $\alpha_{T} /\left(\alpha_{\mathrm{s}} \alpha_{P}-1\right)<0$ since any density decrease due to expansion will lead to a further temperature increase (see Eq. (4)). However, if the absolute value of $\alpha_{T} /\left(\alpha_{\mathrm{s}} \alpha_{P}-1\right)$ is large enough, a small increase in temperature induces such a large decrease in density that the nuclear energy generation may be considerably reduced. In this
$\mathrm{Y}=0.66, \mathrm{X}_{\mathrm{C}}=0.28$
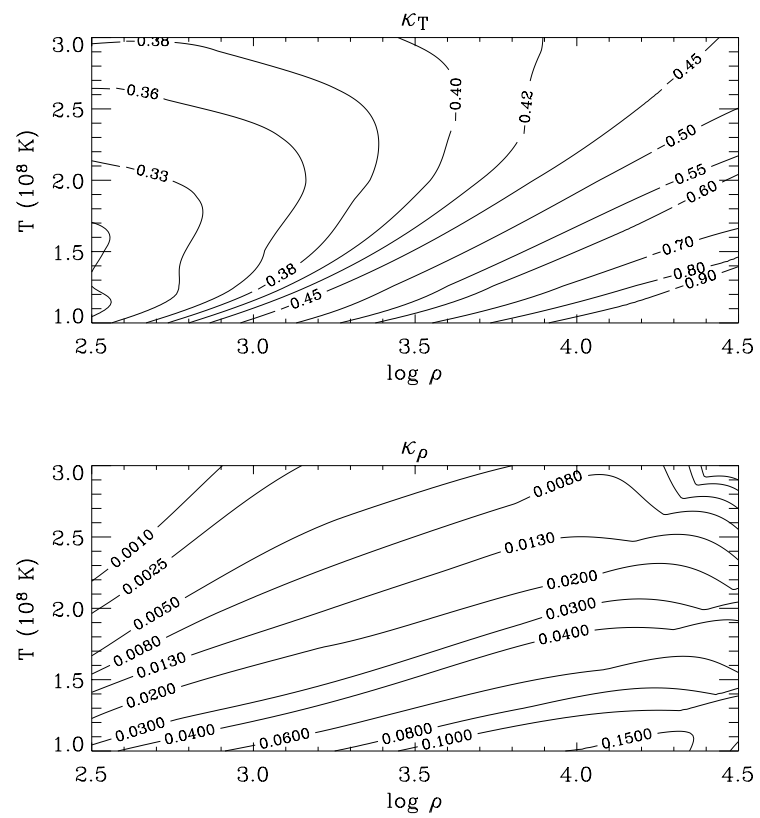

Fig. 4. The logarithmic derivatives of the opacity with respect to to temperature and density: $\kappa_{T}=(\partial \ln \kappa / \partial \ln T)_{\rho}$ and $\kappa_{\rho}=$ $(\partial \ln \kappa / \partial \ln \rho)_{T}$. The OPAL opacity table by Iglesias \& Rogers (1996) has been used in the calculation. $Y=0.662, X_{\mathrm{C}}=0.286$ and $Z=0.02$ have been assumed.

case, we have $\sigma_{\mathrm{E}}<0$ and the shell nuclear burning is thermally stable.

This can be realized when $\alpha_{T}$ becomes very large or/and when $\left|\alpha_{\mathrm{s}} \alpha_{P}-1\right| \ll 1$. A large $\alpha_{T}$ can be obtained if radiation pressure becomes substantial (Fig. 1c), showing again its stabilizing effect. When $\left|\alpha_{\mathrm{s}} \alpha_{P}-1\right| \ll 1$ is satisfied, we have $\delta \rho / \rho=\frac{1}{\alpha_{\mathrm{s}}} \delta P / P \simeq \alpha_{P} \delta P / P$. This means that the shell source behaves as if it were isothermal when $\alpha_{\mathrm{s}} \alpha_{P} \simeq 1$. As a consequence, the influence of the temperature perturbation becomes negligible compared to that of the density perturbation, resulting in $\sigma_{\mathrm{E}}<0$. For an ideal gas with $\beta=1$, this condition means $\alpha_{\mathrm{s}} \simeq 1$ : the shell source is stable if its thickness $D$ is not much less than $0.37 r_{\mathrm{s}}$, even when $c^{*}>0$ (see Fig. 5). This mode of stability has been already noted by Fujimoto (1982b): he found that a very large $c^{*}$ can render a shell source stable, which is the case when $\left|a_{\mathrm{s}} a_{P}-1\right| \ll 1$ (Eq. (6)). As shown in Sect. 5, this situation may occur in the helium shell sources of AGB stars and accreting white dwarfs.

\subsection{Infinitely thin shell sources}

If a shell source is infinitely thin, we have $\alpha_{\mathrm{s}} \rightarrow 0, c^{*} \rightarrow c_{P}$ and

$\sigma=v-4+\kappa_{\mathrm{T}}-\alpha_{T}\left(\lambda+\kappa_{\rho}\right)$.

The shell burning can be stable if the dependence of the nuclear reaction on temperature is weak or/and when radiation pressure is significant, in which case $\alpha_{T}$ becomes large (cf. Fig. 5). An astrophysical application for such a case is shell burning in accreting neutron stars, for which the compactness of the gravitating body enforces a constant pressure in the shell source, 


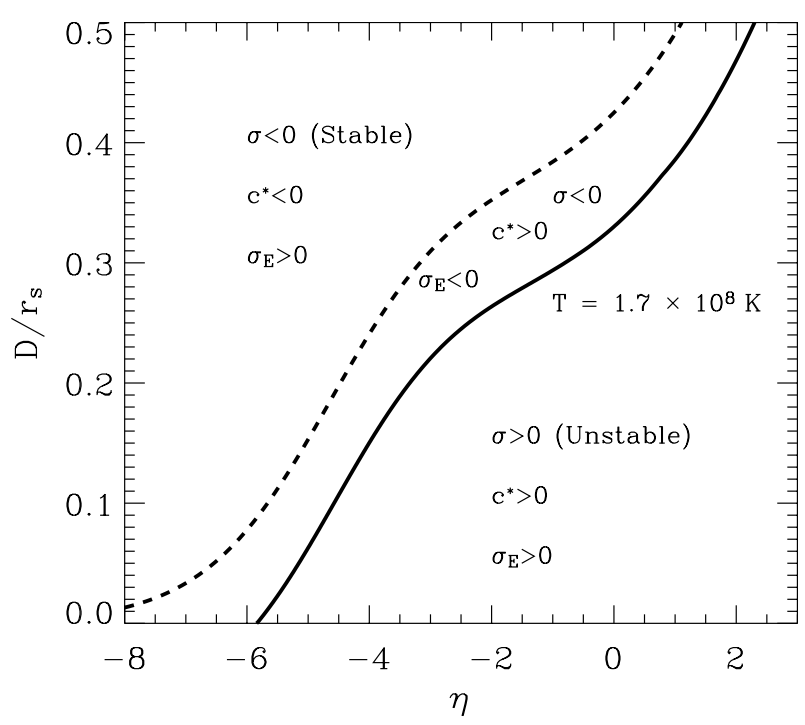

Fig. 5. Loci of $\sigma$ and $c^{*}$ for their sign in $\eta-D / r_{\mathrm{s}}$ plane when $T=$ $1.7 \times 10^{8} \mathrm{~K}$. The solid line separates the thermally unstable $(\sigma>0)$ from the stable $(\sigma<0)$ region, while the dashed line separates the region with negative $c^{*}$ from that with positive $c^{*}$. The same chemical composition as in Fig. 4 is assumed.

such that $\alpha_{\mathrm{s}} \rightarrow 0$. In fact, the criterion for thermal instability in this case is very similar to that given by Fujimoto et al. (1981), who studied shell flashes on accreting neutron stars. Numerical calculations by Taam et al. (1996) show that the helium shell burning in a neutron star can be stable when the temperature of the helium shell source is as high as $5.6 \times 10^{8} \mathrm{~K}$, for which the temperature sensitivity of the $3 \alpha$ reaction is considerably weakened ( $v \simeq 5$, Fig. 3 ). This can be easily understood by our stability criterion with Eq. (24) (see also Fig. 7 and discussions given in Sect. 4).

\subsection{Complete degeneracy}

In completely degenerate gas $(\eta \rightarrow \infty)$, we have $\alpha_{T}=0$ and $\sigma$ becomes

$\sigma=v-4+\kappa_{T}$.

Since heat in a strongly degenerate gas is transfered mainly by electron conduction, with $\kappa_{T} \simeq 2$ (e.g. Kippenhahn \& Weigert 1990), any nuclear reaction with $v>2$ will give $\sigma>0$, resulting in unstable nuclear burning. An example for the shell burning in strongly degenerate conditions is provided by the sub-Chandrasekhar mass progenitor model for Type Ia supernovae. In this model, helium ignites at the bottom of a helium layer of some tenths of a solar mass accumulated on a CO white dwarf with low accretion rates (e.g. Woosley \& Weaver 1994). At the ignition point, the helium envelope is highly degenerate $(\eta>10$, e.g. Yoon \& Langer 2004), rendering complete degeneracy a good approximation.

\section{Instability diagrams}

Figure 5 gives an example of the stability criterion for a $\mathrm{He}$ shell source in $\eta-D / r_{\mathrm{s}}$ parameter space, using a specific chemical composition as indicated in the figure caption, which is typical for helium shell sources in AGB stars or in accreting white dwarfs.

This figure shows the two modes of stability as discussed in Sects. 3.1 and 3.2: one with $c^{*}<0$ and $\sigma_{\mathrm{E}}>0$ and the other with $c^{*}>0$ and $\sigma_{\mathrm{E}}<0$. As expected, a shell source becomes more susceptible to thermal instability when it is thinner and more degenerate. For $\eta \gtrsim 2$, where the electron degeneracy is significant $(F(\eta) \gtrsim 1.5)$, the shell source cannot be stable even with $D / r_{\mathrm{s}}>0.5$, which is because a pressure change becomes less sensitive to a temperature change as the electrons become more degenerate. On the other hand, the shell source can be stable even with $D / r_{\mathrm{s}}=0$ when $\eta \lesssim-5.8$ (see also Fig. 7), where the radiation pressure is dominant over the gas pressure.

As shown in Fig. 4, $\kappa_{T}$ and $\kappa_{\rho}$ remain small compared to $v$ in the given temperature and density range. The quantities $\alpha_{P}$ and $\alpha_{T}$ do not depend strongly on $\mu_{\mathrm{e}} / \mu_{\mathrm{I}}$. The main factor which determines the stability at given $\eta$ and $D / r_{\mathrm{s}}$ is thus the temperature of the shell source. Figure 6 shows that the stability criterion is more relaxed for higher temperature. This is because the temperature dependence of the energy generation is weakened (Fig. 3), and because radiation pressure becomes more significant at a given degree of degeneracy as the temperature increases. This temperature dependence of the stability of shell burning explains why the accretion rates which allow steady burning solutions depend on the mass of the accreting white dwarf (e.g. Fujimoto 1982b; Nomoto \& Kondo 1991; Cassisi et al. 1998). I.e., higher accretion rates are required to have stable shell burning for more massive white dwarfs, in which shell sources are thinner. This is mainly because shell sources becomes hotter with higher accretion rates if the accreted matter is burned in a steady way.

In Fig. 7, we show the stability conditions in the densitytemperature plane for 5 different relative shell source thicknesses. As discussed in Sect. 3.3, this figure shows that a shell source can be stable even when it is infinitely thin $\left(D / r_{\mathrm{s}}=0\right)$, if the temperature is high enough, since with high temperature the dependence of the energy generation on temperature becomes weakened and the role of radiation pressure becomes important, giving a large $\alpha_{T}$. The stability conditions are relaxed as $D / r_{\mathrm{s}}$ increases. The conditions for stability in hydrogen shell sources show a similar behaviour in Fig. 8, where the shell source is assumed to be powered by the CNO cycle. I.e., thinner, cooler and more degenerate shell sources are more prone to thermal instability. Note, however, that in the calculation we did not consider the so called hot $\mathrm{CNO}$ cycle, which may be active at high temperatures $\left(T>8 \times 10^{7} \mathrm{~K}\right)$.

\section{Applications}

In this section we apply the stability criterion to various numerical stellar models: a $3.0 M_{\odot}$ AGB star, helium accreting $\mathrm{CO}$ white dwarfs, a cooling helium white dwarf with a thin hydrogen envelope, and a $1.0 M_{\odot}$ giant. All models have been computed with a stellar evolution code (Langer 1998, and references therein) which solves the hydrodynamic form of the stellar structure equations (Kippenhahn \& Weigert 1990). Opacities are taken from Iglesias \& Rogers (1996). We define 


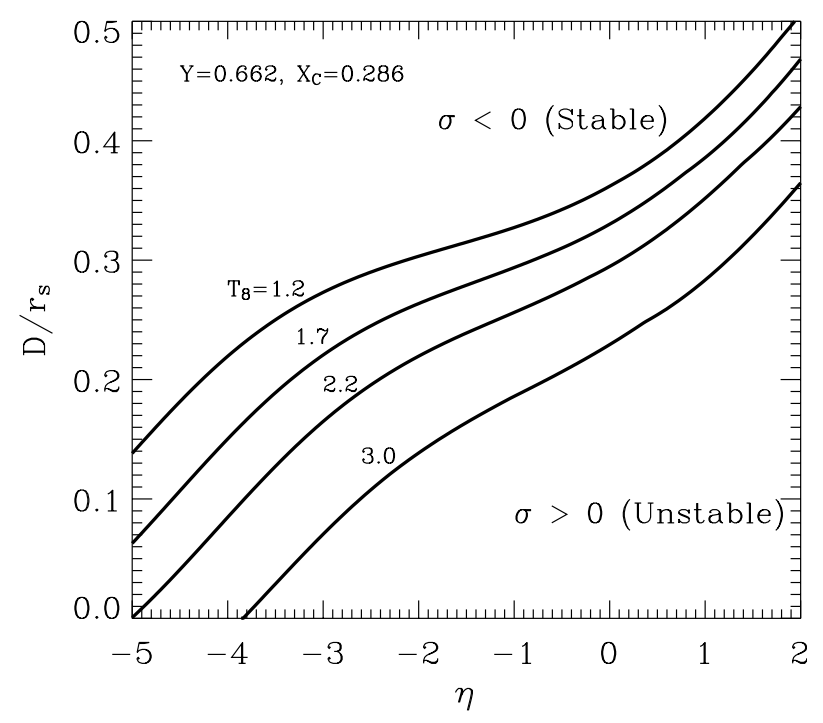

Fig. 6. Loci for the sign of $\sigma$ at 4 different temperatures: $T=1.2,1.7,2.2$ and $3.0 \times 10^{8} \mathrm{~K}$ in $\eta-D / r_{\mathrm{s}}$ plane.

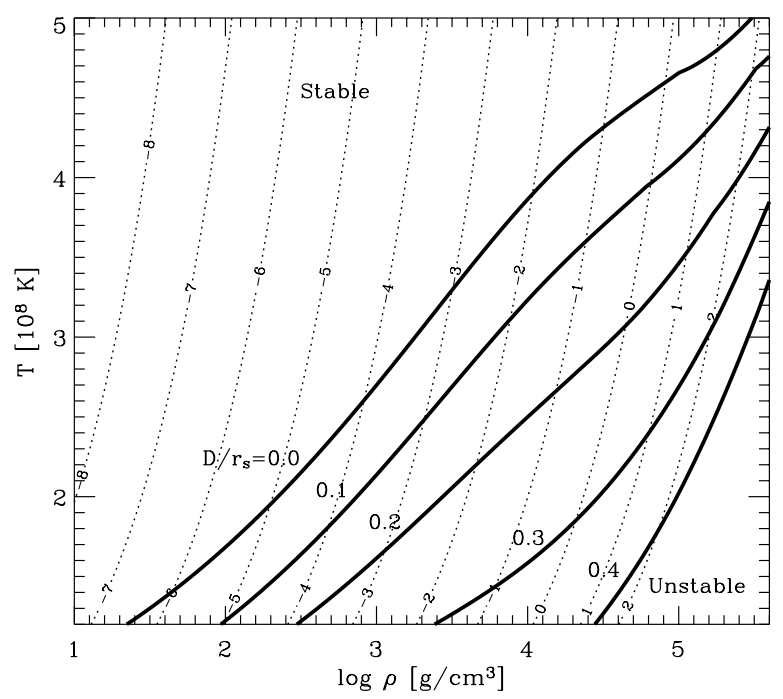

Fig. 7. Stability conditions for a helium shell source in the densitytemperature plane. The solid line separates the thermally unstable $(\sigma>0$, lower right part) from the stable $(\sigma<0)$ region, for 5 different relative shell source thicknesses (i.e., $D / r_{\mathrm{s}}=0.0,0.1,0.2,0.3$ and 0.4). The dotted contour lines denote the degeneracy parameter $\eta$. $X_{\mathrm{He}}=0.662, X_{\mathrm{C}}=0.280$ and $Z=0.02$ have been assumed.

the thickness of a shell source $D$ such that the energy generation rate at each boundary has a certain fraction $\left(:=f_{\mathrm{b}}\right)$ times its peak value. Values of $D / r_{\mathrm{s}}$ in stellar models would be affected by different choices of $f_{\mathrm{b}}$. For instance, $f_{\mathrm{b}}=10^{-3}$ results in a larger $D / r_{\mathrm{s}}$ by about $15 \%$ compared to the case of $f_{\mathrm{b}}=10^{-2}$, in helium accreting white dwarf models presented in Sect. 5.2. In our applications, we choose $f_{\mathrm{b}}=2 \times 10^{-3}$ because this particular choice gives the best prediction for the onset of thermal instability of shell burning for all different types of stellar models discussed below. The mean values weighted by the energy generation rate over the shell source are used for density, temperature and chemical abundances to estimate physical quantities which appear in Eq. (12).

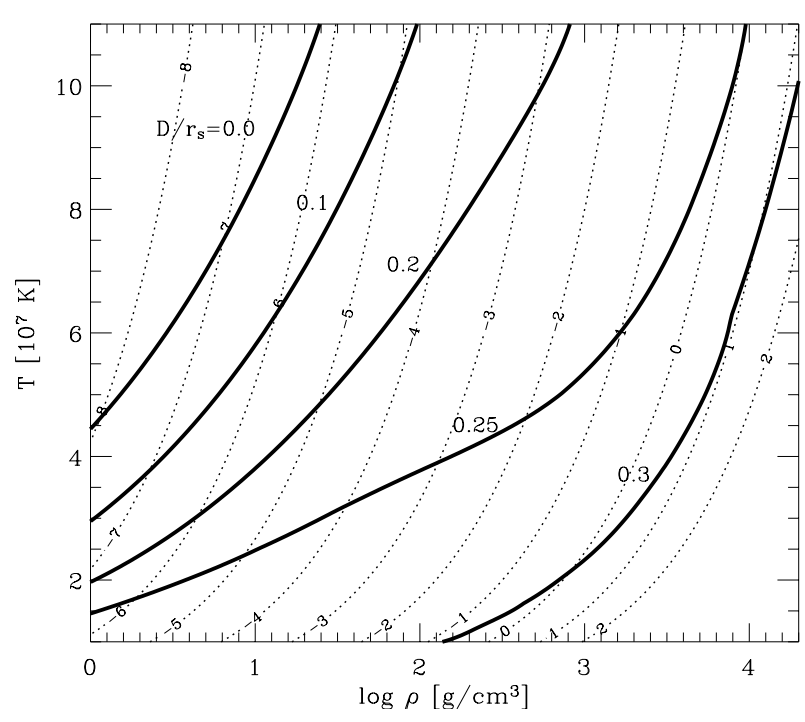

Fig. 8. Stability conditions for the hydrogen shell source powered by the $\mathrm{CNO}$ cycle, in density and temperature plane. The solid line separates the thermally unstable $(\sigma>0)$ from stable $(\sigma<0)$ region at 5 different relative thicknesses of shell source (i.e., $D / r_{\mathrm{s}}=0.0,0.1,0.2$, 0.25 and 0.3 ). A shell source with each $D / r_{\mathrm{s}}$ is expected to be stable in the upper region of each locus. The dotted contour lines denote the degeneracy parameter $\eta . X_{\mathrm{H}}=0.502, X_{\mathrm{He}}=0.478$ and $Z=0.02$ have been assumed. Note that the hot $\mathrm{CNO}$ cycle is not considered in the calculation.

\subsection{Helium shell burning on the AGB}

It is well known that asymptotic giant branch (AGB) stars suffer thermally unstable helium shell burning in their advanced evolution (e.g. Iben \& Renzini 1983). We investigate this phase by computing a $3 M_{\odot}$ evolutionary model with $Z=0.02$, starting at the zero age main sequence (Langer et al. 1999). After passing through hydrogen and helium core burning, the star enters the helium shell burning stage. Figure 9a shows the nuclear luminosity due to helium burning as a function of time at the onset of the thermal pulses ( $T \simeq 4.306 \times 10^{8} \mathrm{yrs}$ ). We analyzed those models indicated by a filled circle in Fig. 9a, in which the helium shell burning is still stable, and computed $c^{*}$ and $\sigma$ for each model. (Figs. 9b and 9c). During the stable shell burning stage, the mean temperature in the shell source is nearly constant at $T \simeq 1.4 \times 10^{8} \mathrm{~K}$ (Fig. 9d). The shell source becomes thinner and more degenerate as the $\mathrm{CO}$ core mass increases, as shown in Fig. 9e.

The gravothermal specific heat of the shell source is positive in the chosen models, indicating that the shell burning is kept stable until the onset of the thermal pulses by the second mode of the stability discussed in Sect. 3.2. However, it becomes smaller as the $\mathrm{CO}$ core mass increases, mainly because $\alpha_{\mathrm{s}} \alpha_{P}-1$ deviates from 1 more significantly as $D / r_{\mathrm{s}}$ decreases (see Eq. (6)), and partly because degeneracy in the shell source becomes stronger (Fig. 9e). The thermal pulses start when the relative thickness of the shell source becomes as low as 0.316 . 


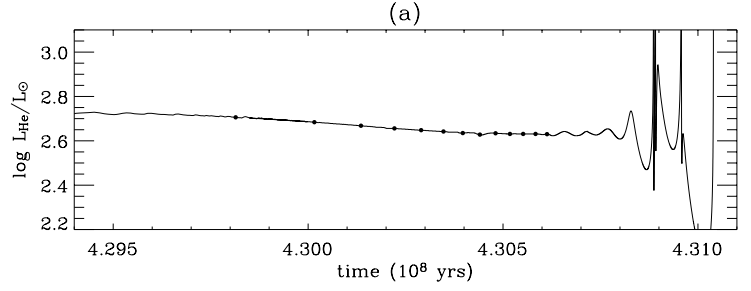

(b)
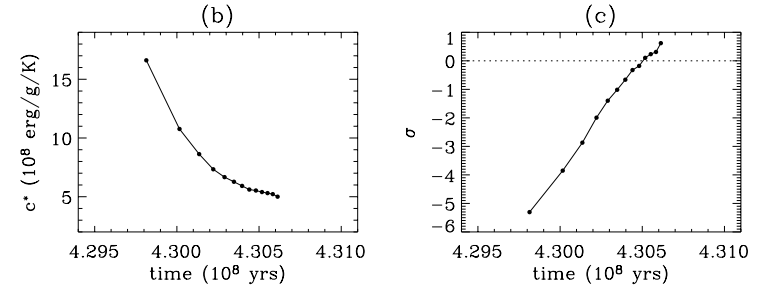

(d)
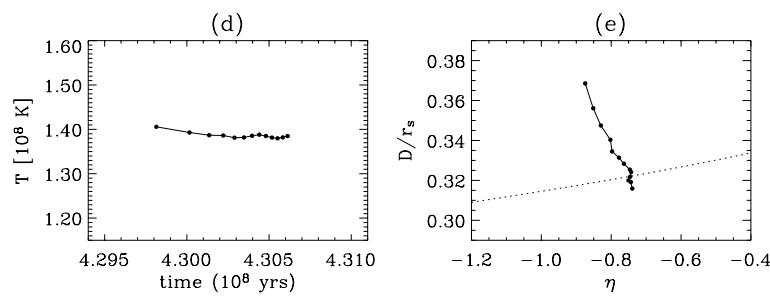

Fig. 9. Evolution of the helium shell source in a $3 M_{\odot}$ AGB star. a) Nuclear luminosity due to helium burning as a function of time, for shortly before and during the onset of thermal pulses. The mean values of $c^{*}, \sigma$, and temperature in the shell sources of the models marked by a filled circle are shown in $\mathbf{b}), \mathbf{c})$ and d) respectively. e) Evolution of the shell source in the $\eta-D / r_{\mathrm{s}}$ plane. The dotted line corresponds to $\sigma=0$ computed for the physical conditions in the shell source of the last analyzed model.

\subsection{Helium shell burning in accreting white dwarfs}

Here we consider the helium shell source in hot heliumaccreting CO white dwarfs. Two different initial white dwarf models are considered: $M_{\text {init }}=0.8 M_{\odot}$ with $\log L_{\mathrm{s}} / L_{\odot}=2.948$ and $M_{\text {init }} 0.998 M_{\odot}$ and $\log L_{\mathrm{s}} / L_{\odot}=3.379$, where $M_{\text {init }}$ and $L_{\mathrm{s}}$ are the initial mass and surface luminosity, respectively. The accreted matter is assumed to be helium-enriched such that $Y=0.98$. We have performed simulations with 6 different combinations of initial mass and accretion rate (see Table 1). The helium shell burning is initially stable in each model sequence, and eventually turns unstable as the white dwarf mass increases (see Fig. 10 for an example). The value of $\sigma$ for the shell source of the last analyzed model in each sequence is given in the last column of the table. In all sequences, the last analyzed models, from which the thermal pulses grow significantly, have $|\sigma| \lesssim 0.6$, indicating that our stability criterion predicts the onset of the instability with good accuracy.

As an example, results for sequence No. 6 are shown in Fig. 10. In Fig. 10a, the nuclear luminosity due to helium burning is given as a function of the total white dwarf mass, which serves as a linear measure of time. Thermal pulses are induced when the white dwarf mass reaches $\sim 1.028 M_{\odot}$. The evolution of $c^{*}$ and $\sigma$ shown in Figs. $10 \mathrm{~b}$ and $10 \mathrm{c}$ is very similar to that in the AGB model discussed above. The mean temperature in the shell source is about $2 \times 10^{8} \mathrm{~K}$ (Fig. 10d). The shell source
Table 1. Properties of the computed helium accreting white dwarf models. The first column denotes the model sequence number. The second column shows the initial mass and the third gives the accretion rate. The fourth column gives the amount of accreted material until the onset of the helium shell instability. The value of $\sigma$ estimated in the shell source of the last analyzed model is given in the last column.

\begin{tabular}{ccccc}
\hline \hline Nos. & $M_{\text {init }}\left(M_{\odot}\right)$ & $\dot{M}\left(M_{\odot} \mathrm{yr}^{-1}\right)$ & $\Delta M\left(M_{\odot}\right)$ & $\sigma$ \\
\hline No. 1 & 0.800 & $4.0 \times 10^{-7}$ & 0.0212 & -0.429 \\
No. 2 & 0.800 & $5.0 \times 10^{-7}$ & 0.0238 & -0.291 \\
No. 3 & 0.800 & $7.0 \times 10^{-7}$ & 0.1141 & -0.598 \\
No. 4 & 0.800 & $8.0 \times 10^{-7}$ & 0.1455 & -0.471 \\
No. 5 & 0.998 & $8.0 \times 10^{-7}$ & 0.0028 & -0.114 \\
No. 6 & 0.998 & $1.0 \times 10^{-6}$ & 0.0048 & -0.035 \\
\hline
\end{tabular}

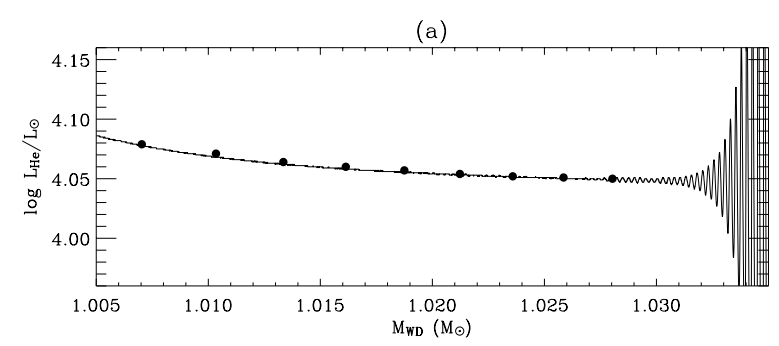

(b)

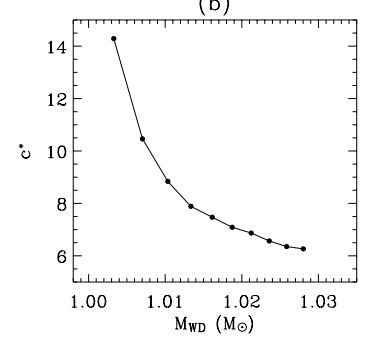

(c)

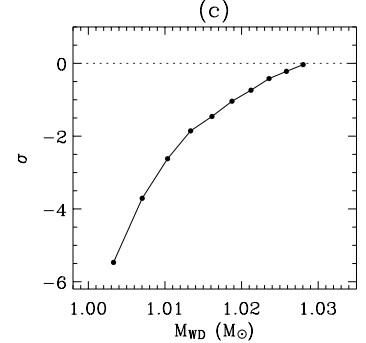

(d)
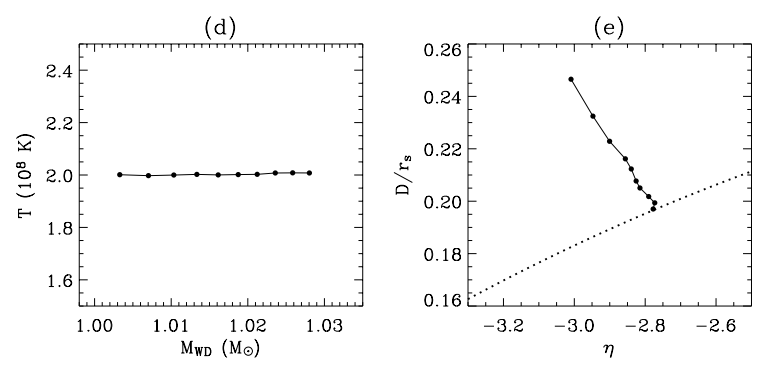

Fig. 10. Evolution of the helium shell source in an accreting white dwarf with an initial mass of $0.998 M_{\odot}$ and a constant accretion rate of $10^{-6} M_{\odot} \mathrm{yr}^{-1}$. a) Nuclear luminosity due to helium burning as a function of the total white dwarf mass. The mean values of $c^{*}, \sigma$, and temperature in the shell sources of the models marked by a filled circle are shown in b), c) and d) respectively. e) Evolution of the shell source in the $\eta-D / r_{\mathrm{s}}$ plane. The dotted line corresponds to $\sigma=0$ computed for the physical conditions in the shell source of the last analyzed model.

becomes thinner and more dense as the white dwarf mass increases (Fig. 10). The last analyzed model is at the edge of stability as shown in Fig. 10c and the thermal pulses grow significantly from this point. 

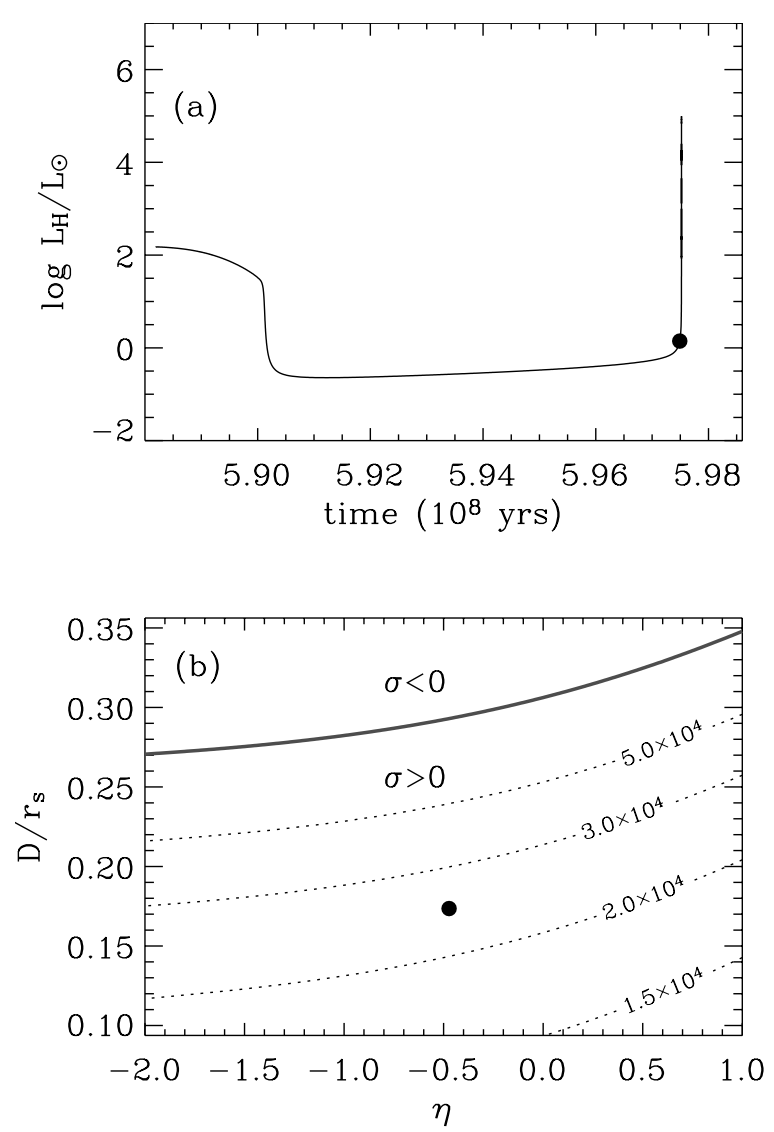

Fig. 11. a) Nuclear luminosity due to hydrogen burning at the onset of the hydrogen shell flash in the hydrogen envelope in a cooling helium white dwarf of $0.324 M_{\odot}$. b) Contour levels of the perturbation growth time scale in the $\eta-D / r_{\mathrm{s}}$ plane, obtained from Eq. (13). Here, $\sigma$ and $\tau_{\text {th }}$ have been estimated for the physical conditions in the shell source of the marked model. The solid line corresponds to $\sigma=0$.

\subsection{Hydrogen shell burning in cooling helium white dwarfs}

Here we discuss a hydrogen shell flash in a cooling helium white dwarf model (cf., Driebe et al. 1999), which has been obtained by evolving a $2.4 M_{\odot}$ zero age main sequence star with $Z=0.02$ in a binary system with an initial orbital period of 1.76 days. Its companion was a $2 M_{\odot}$ zero age main sequence star. It loses most of the hydrogen-rich envelope during the hydrogen shell burning stage through so-called case B mass transfer, ending in a helium white dwarf of $M=0.324 M_{\odot}$ with a hydrogen envelope of $M \simeq 0.005 M_{\odot}$ when the mass transfer stops. The luminosity and surface temperature at this moment are $158.5 L_{\odot}$ and $1.4 \times 10^{4} \mathrm{~K}$, respectively.

The hydrogen shell burning is weakened progressively as the white dwarf cools (Fig. 11a). At $t \simeq 5.905 \times 10^{8} \mathrm{yr}$, further weakening of the shell burning stops due to the increase in density. The hydrogen burning increases slowly as the helium core contracts further and ends in a strong flash at $t \simeq 5.98 \times 10^{8} \mathrm{yr}$. The thickness and degree of degeneracy of the shell source have been computed for the model marked by a filled circle in Fig. 11a, and positioned in the $D / r_{\mathrm{s}}-\eta$ plane in Fig. 11b. The corresponding temperature and density of the

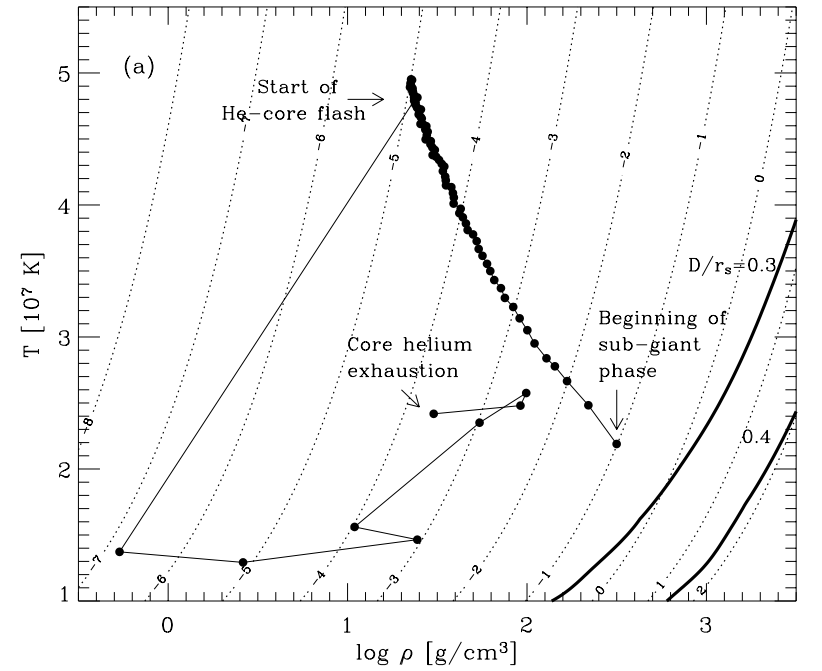

(b)
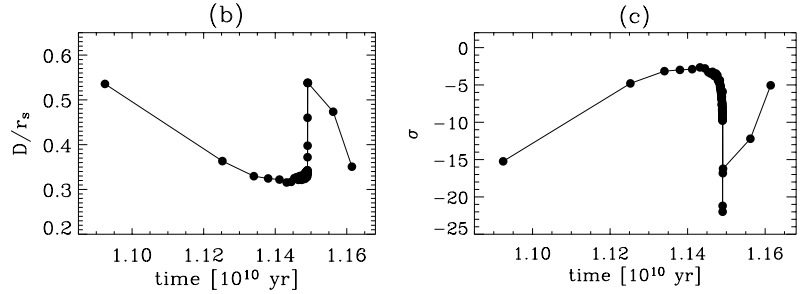

Fig. 12. Evolution of a 1.0 star with solar metalicity from the beginning of the sub-giant phase until the exhaustion of core helium burning. a) Evolution of the hydrogen shell source in the density - temperature plane. The thick solid lines correspond to $\sigma=0$ for $D / r_{\mathrm{s}}=$ 0.3 and 0.4 (cf. Fig. 8). The evolutionary stages at different epochs are indicated with short arrows. The dotted contour lines give the degeneracy parameter $\eta$. b) The relative thickness of the shell source as a function of time. c) The value of $\sigma$ in the shell source as a function of time.

shell source are $2.13 \times 10^{7} \mathrm{~K}$ and $6.91 \times 10^{2} \mathrm{~g} \mathrm{~cm}^{-3}$, respectively. The shell source at this point is in the unstable regime as expected from the fact that the nuclear luminosity reaches its peak very soon thereafter. The contour levels for the perturbation growth timescale from Eq. (13) are also given in the same figure. The perturbation growth time estimated at the point is about $2.4 \times 10^{4} \mathrm{yrs}$, which is in good agreement with the time interval from the marked model to the peak in $L_{\mathrm{H}}\left(\simeq 3 \times 10^{4} \mathrm{yrs}\right)$.

\subsection{Hydrogen shell burning in a $1.0 M_{\odot}$ giant}

It is a matter of debate whether shell flashes in hydrogen shell sources of low-mass sub-giants and giants could be a possible cause for anomalies of chemical abundances in stars of globular clusters (e.g. Denissenkov \& van den Berg 2003). Stellar evolution models indicate that hydrogen shell sources in subgiants or red giants are usually thermally stable. Here, we analyzed the physical conditions of the hydrogen shell source in a $1.0 M_{\odot}$ model with solar metallicity.

Figure 12a shows the evolution of the hydrogen shell source in the density-temperature plane, from the beginning of the sub-giant phase until the core helium exhaustion. Note that, from the beginning of the sub-giant until the core helium flash, 
the shell source becomes hotter and less dense (Fig. 12a), while the relative thickness of the shell source remains larger than 0.3 (Fig. 12b). Once the core helium flash occurs, both the temperature and density of the shell source decrease rapidly due to the expansion. Throughout the considered stages, the evolutionary track of the shell source remains in the thermally stable regime (cf. Fig. 12c). This result indicates that the onset of the thermal instability in hydrogen shell sources of low-mass subgiants and giants is unlikely.

\section{Conclusion}

We have developed a stability criterion for thermonuclear shell sources which can be easily applied to numerical stellar models in a quantitative way. The stabilizing/destabilizing factors have been identified unambiguously: a shell source is less prone to the thermal instability when it is thicker, less degenerate and hotter (Sect. 3, Figs. 6-8). If a shell source is sufficiently thick at a given degree of degeneracy, the gravothermal specific heat becomes negative and any excessive energy gain is consumed mostly for the expansion work, preventing thermal runaway. It is shown that the shell source can remain stable even with a positive gravothermal specific heat as long as the temperature at a given degree of degeneracy is high enough such that the sensitivity of nuclear reactions to temperature is significantly weakened or/and that radiation pressure becomes substantial. In such a case, the energy loss rate by radiation begins to dominate over the additional nuclear energy production rate. Interestingly, this effect allows even an infinitely thin shell source to be thermally stable (Sect. 3.3, Figs. 7 and 8), which may have interesting consequences in accreting neutron stars (Sect. 3.3; cf. in 't Zand et al. 2003).

We have applied the stability criterion developed in this study to a $3 M_{\odot}$ AGB star, to helium accreting $\mathrm{CO}$ white dwarfs, to a helium white dwarf with a thin hydrogen shell, and to a $1.0 M_{\odot}$ giant (Sect. 5). In spite of the simple approach of using homology assumption in the shell source and not relying on the full linear stability analysis, it is demonstrated that this criterion can predict the onset of thermal pulses or flashes with a reasonably good accuracy.

In conclusion, being simple and robust, our stability criterion may serve as a useful tool for analyzing shell burning in various types of stars. One example can be found in Yoon et al. (2004), who discuss the effects of rotationally induced chemical mixing on the behaviour of helium shell burning in accreting white dwarfs.
Acknowledgements. We are grateful to the anonymous referee for fruitful and enlightening comments which led to improvement and expansion of the text. This research has been supported in part by the Netherlands Organization for Scientific Research (NWO).

\section{References}

Busso, M., Roberto, G., Lambert, D., Travaglio, C., \& Smith, V. V. 2001, ApJ, 557, 802

Cassisi, S., Iben, I., \& Tornambé, A. 1998, ApJ, 496, 376

Clayton, D. 1968, Principles of Stellar Evolution and Nucleosynthesis (Chicago)

Denissenkov, P. A., \& van den Berg, D. A. 2003, ApJ, 598, 1246

Dennis, T. R. 1971, ApJ, 167, 311

Driebe, T., Blocker, T., Schönberner, D., \& Herwig, F. 1999, A\&A, 350, 89

Fujimoto, M. Y. 1982a, ApJ, 257, 752

Fujimoto, M. Y. 1982b, ApJ, 257, 767

Fujimoto, M. Y., Hanawa, T., \& Miyaji, S. 1981, ApJ, 246, 267

Giannone P., \& Weigert, A. 1967, Z. Astrophys., 67, 41

Härm, R., \& Schwarzshild, M. 1972, ApJ, 172, 403

Harris, M. J., Fowler, W. A., Caughlan, G. R., \& Zimmerman, B. A. 1983, ARA\&A, 21, 165

Huang, R. Q., \& Yu, K. N. 1998, Stellar Astrophysics (Springer-Verlag)

Iben, I., \& Renzini, A. 1983, ARA\&A, 27, 271

Iglesias, C. A., \& Rogers, F. J. 1996, ApJ, 464, 943

Kippenhahn, R., \& Weigert, A. 1990, Stellar Structure and Evolution (Springer-Verlag)

Langer, N. 1998, A\&A, 329,551

Langer, N., Heger, A., Wellstein, S., \& Herwig, F. 1999, A\&A, 346, L37

Langer, N., Yoon, S.-C., Wellstein, S., \& Scheithauer, S. 2002, in The Physics of Cataclysmic Variables and Related Objects, ed. B. T. Gänsicke, K. Beuermann, \& K. Reinsch, ASP Conf. Proc., 261, 252

Lugaro, M., Herwig, F., Lattanzio, J. C., Gallino, R., \& Straniero, O. 2003, ApJ, 586, 1305

Nomoto, K., \& Kondo, Y. 1991, ApJ, 367, L19

Sackmann, I. J. 1977, ApJ, 212, 159

Schwarzshild, M., \& Härm, R., 1965, ApJ, 142, 855

Stothers, R., \& Chin, C.-W. 1972, ApJ, 177, 155

Taam, R. E., Woosley, S. E., \& Lamb, D. Q. 1996, ApJ, 459, 271

Unno, W. 1970, PASJ, 22, 299

Weigert, A. 1966, Z. Astrophys., 64, 395

Woosley, S. E., \& Weaver, T. A. 1994, ApJ, 423, 371

Yoon, S.-C., \& Langer, N. 2004, A\&A, 419, 645

Yoon, S.-C., Langer, N., \& Scheithauer, S. 2004, A\&A, 425, 217

in 't Zand, J. J. M., Kuulkers, E., Verbunt, F., Heise, J., \& Cornelisse, R. 2003, A\&A, 411, L487 